\title{
Explorando las conexiones matemáticas asociadas a la función exponencial y logarítmica en estudiantes universitarios colombianos
}

\section{Exploring mathematical connections associated with the exponential and logarithmic function in Colombian university students}

\author{
Karen Gisel Campo-Meneses ${ }^{1}$ \\ Javier García-García
}

\begin{abstract}
Resumen: Las conexiones matemáticas representan una meta en el currículo de diversos países, por lo que han sido objeto de investigación en Matemática Educativa. El presente artículo está inserto en esta línea y busca responder la pregunta ¿Qué conexiones matemáticas realizan los estudiantes universitarios que cursan carreras afines a la matemática cuando resuelven tareas que involucran a las funciones exponencial y logarítmica? Las conexiones matemáticas se entienden como un proceso en el que una persona establece una relación verdadera entre dos o más ideas, conceptos, teoremas, procedimientos y significados entre sí, con otras disciplinas o con la vida real. El estudio emplea a la Entrevista Basada en Tareas (previamente validadas) para la colecta de datos, los cuales fueron analizados utilizando el análisis temático. Los resultados obtenidos indican la aparición de seis tipologías de conexiones matemáticas: parte-todo, característica, significado, procedimental, reversibilidad y representaciones
\end{abstract}

Fecha de recepción: 01 de agosto de 2019. Fecha de aceptación: 15 de octubre de 2020.

1 Universidad Autónoma de Guerrero, Centro de Investigación en Matemática Educativa, Chilpancingo de los Bravo, Gro, México. karencampo@uagro.mx, orcid.org/0000-0001-7483-3134

2 Universidad Autónoma de Guerrero, Centro de Investigación en Matemática Educativa, Chilpancingo de los Bravo, Gro, México. jagarcia@uagro.mx, orcid.org/0000-0003-4487-5303 
diferentes y una inclinación, por parte de los estudiantes, para dar respuesta a las tareas que se asocian con la función exponencial.

Palabras clave: conexiones matemáticas, función exponencial, función logarítmica, Entrevista Basada en Tareas, estudio de casos.

\begin{abstract}
Mathematical connections are a goal in the curriculum of several countries, so they have been the subject of research in Educational Mathematics. This article is inserted in this line and seek to answer the question: What mathematical connections do university students who study careers related to mathematics make when they solve tasks that involve the exponential and logarithmic functions? Mathematical connections are understood as a process in which a person establishes a true relationship between two or more ideas, concepts, theorems, procedures and meanings with each other, with other disciplines or with real life. The study uses the Task Based Interview (previously validated) for data collection, the results of which were analyzed using thematic analysis. The results obtained indicate the appearance of six types of mathematical connections: part-whole, characteristic, meaning, procedural, reversibility and different representations and an inclination, on the part of the students, to respond to the tasks that are associated with the exponential function.
\end{abstract}

Keywords: mathematical connections, exponential function, logarithmic function, Task-Based Interview, study of cases.

\title{
1. INTRODUCCIÓN
}

Las investigaciones en Matemática Educativa revelan la importancia de realizar conexiones matemáticas -concepto que tuvo auge desde que fue incorporado en los estándares curriculares por el National Council of Teachers of Mathematics (NCTM, 1989) en el currículum norteamericano- en el proceso de enseñanza- aprendizaje. Las conexiones matemáticas, en la literatura en Matemática Educativa, son entendidas como un proceso en el que una persona establece una relación verdadera entre dos o más conceptos, ideas, teoremas, procedimientos o significados entre sí, con la vida real o con otras disciplinas (García-García y Dolores-Flores, 2018). Su importancia estriba según Eli et al. (2013), en que 
cuando los estudiantes conectan ideas matemáticas su comprensión es más profunda y duradera, lo que les permite ver a las matemáticas como un todo coherente e integrado. Además, cuando realizan conexiones matemáticas es evidencia de que le han encontrado utilidad a la matemática.

Una revisión de la literatura permite identificar que el estudio de las conexiones matemáticas tiene al menos cuatro orientaciones (García, 2018; García-García, 2019): (1) aquella que estudia las conexiones matemáticas centradas en un concepto matemático particular utilizando ciertas tareas matemáticas (Bingölbali y Coşkun, 2016; Brutlag y Maple, 1992; Dolores y García-García, 2017; Dolores-Flores et al., 2019; Eli et al., 2011, 2013; Jaijan y Loipha, 2012; Koestler et al., 2013 y Mhlolo, 2012); (2) aquella que estudia las conexiones matemáticas entre diferentes representaciones (Elia et al., 2007; Moon et al., 2013; Noss et al., 1997); (3) la que estudia las conexiones matemáticas que aparecen cuando estudiantes o profesores resuelven problemas de aplicación o modelado (Baki et al., 2009; Cawley y Foley, 2002; Glass, 2002; Frykholm y Glasson, 2005; Gainsburg, 2008; Hurst, 2007; Yoon et al., 2010); (4) la que estudia las creencias asociadas al uso de las conexiones matemáticas en situación escolar (Ji-Eun, 2012).

Entre otros resultados, las investigaciones previas indican que las conexiones matemáticas son un camino para lograr la comprensión matemática ${ }^{3}$ (Brutlag y Maple, 1992; Cawley y Foley, 2002; Eli et al., 2013; Gainsburg, 2008; Glass, 2002). Así, analizar las conexiones matemáticas que realice un estudiante, permitiría inferir su nivel de comprensión (Mhlolo, 2012). La literatura también indica que estudiantes y profesores usan conexiones matemáticas de diversas tipologías -como procedimental, representaciones diferentes, característica, parte-todo, etc.- cuando resuelven tareas matemáticas y en el proceso de enseñanza (Businskas, 2008; Dolores y García-García, 2017; Evitts, 2004; García, 2018; García-García y Dolores-Flores, 2018, 2019, 2020; Mhlolo, 2012). Asimismo, autores como Bingölbali y Coşkun (2016) y Baki et al. (2009) consideran que acrecentar la habilidad de hacer conexiones matemáticas puede contribuir al desarrollo de las habilidades de razonamiento y comunicación.

Por otra parte, tanto la investigación como el currículum indican que estudiar las funciones exponenciales y logarítmicas es importante por la trascendencia de

3 En este escrito asumimos que la comprensión matemática tiene que ver con la capacidad que tiene el estudiante para usar el contenido matemático para resolver diversas tareas matemáticas. Esto implica que él sea capaz de reconocer propiedades, representaciones y características de un concepto, relacionarlo con otros conceptos matemáticos restantes y usarlo en diferentes situaciones (Font, 2007), es decir, cuando él sea capaz de usar consistentemente un concepto matemático y establecer conexiones matemáticas. 
estos conceptos matemáticos en sí mismos, así como para entender ideas matemáticas más avanzadas o para modelar situaciones de la vida real y de otras ciencias (por ejemplo, la relación entre presión y altura, los procesos radiactivos simples, la reproducción de una colonia de bacterias, algunos crecimientos o decrecimientos demográficos, la inflación, etc.). En ese sentido, el currículo colombiano (Ministerio de Educación Nacional, 2006) muestra que son objeto de estudio tanto en la secundaria como en los cursos de matemáticas (como Cálculo Diferencial o Matemática Fundamental) en la universidad. Su estudio se considera importante porque permite el desarrollo del pensamiento variacional al trabajar con diferentes representaciones distinguiendo su variación como una no lineal.

Entre las investigaciones referentes a las funciones exponencial y logarítmica, se encuentran aquellas que las abordan por separado o las que consideran a ambas de manera articulada. En relación con la función exponencial, se han identificado dos enfoques para su estudio: la teoría de registros de representación semiótica (Sureda y Otero, 2013; Castro et al., 2017) y el razonamiento covariacional (Ellis et al., 2015; Ellis et al., 2016; Trejo y Ferrari, 2018). Mientras que, la función logarítmica ha sido estudiada desde los siguientes enfoques: el conocimiento del profesor (Escobar, 2014) y la comprensión de estudiantes (Chua y Wood, 2005; Gruver, 2018), entre otros. Por otra parte, las investigaciones que han atendido a estas funciones de manera articulada lo han hecho desde la teoría APOE (Weber 2002) y desde el enfoque de razonamiento covariacional (Ferrari-Escolá et al., 2016; Kuper y Carlson, 2020).

En resumen, la revisión de la literatura educativa muestra que es escasa la investigación sobre las funciones exponencial y logarítmica desde el enfoque de conexiones matemáticas. En ese sentido, es importante y pertinente realizar una investigación con esta orientación por las siguientes razones:

- Las conexiones matemáticas son un tema importante en el currículum de diversos países. Además, es vigente en la agenda de investigación en la literatura internacional en el campo de la Matemática Educativa.

- Los conceptos de función exponencial y logarítmica son complejos y muchas veces difíciles para su comprensión. No obstante, una forma de afrontar esta dificultad puede ser mediante la enseñanza y aprendizaje de estas desde la idea de conexiones matemáticas.

- Los resultados permitirán valorar si el marco de referencia construido con base en los datos colectados con estudiantes al resolver tareas sobre derivada e integral es útil al involucrar otros conceptos matemáticos (en el marco conceptual, se explica esto). 
- Finalmente, los resultados podrían permitir, en futuros trabajos, el diseño de una secuencia de actividades que ayude a desarrollar en los estudiantes la habilidad de usar conexiones matemáticas y, con ello, posibilitar una mejora en su comprensión matemática asociada a ambas funciones.

Estas razones nos han permitido plantear nuestra pregunta de investigación en los siguientes términos: ¿Qué conexiones matemáticas realizan los estudiantes universitarios que cursan carreras afines a la matemática cuando resuelven tareas que involucran a las funciones exponencial y logarítmica? La razón de elegir a estudiantes que cursan una carrera afín a matemáticas fue porque consideramos que ellos podrían, como parte de su formación profesional, abordar la aplicabilidad de estas funciones, lo cual permitiría identificar las tipologías de conexiones matemáticas que emplean. Además, en un futuro estos podrían ser profesores de matemáticas y las conexiones matemáticas que empleen podrían ser utilizadas en su práctica futura.

\section{MARCO CONCEPTUAL}

\subsection{CONEXIONES MATEMÁTICAS}

En las investigaciones realizadas en Matemática Educativa encontramos diferentes posturas respecto a la definición de conexiones matemáticas, sin embargo, no hay consenso sobre cómo entenderlas. Por ejemplo, para Godino, et al. (2003) implican establecer relaciones entre distintos objetos matemáticos. La postura del NCTM (2007) es consistente con esta caracterización, pero agrega que es un proceso matemático a través del cual los estudiantes pueden ampliar su comprensión de la matemática basándose en conocimientos previos. Para Singletary (2012), una conexión matemática es una relación entre entidades matemáticas o entre estas y aquellas no matemáticas.

Por su parte, Businskas (2008) considera que las conexiones matemáticas son relaciones verdaderas sobre la base de las cuales está estructurada la matemática y son independientes del estudiante, además son las relaciones mediante las cuales los procesos del pensamiento construyen la matemática. De Gamboa y Figueiras (2014) las entienden como una red de enlaces que permiten coordinar nuevos significados. Consistente con esta postura, Eli et al. (2013) asumen que son un enlace en el que se utilizan conocimientos previos o 
nuevos para establecer o fortalecer la comprensión de las relaciones entre ideas matemáticas, conceptos y representaciones.

García-García y Dolores-Flores (2018) recogen las posturas previas, pero centradas en el quehacer de los estudiantes y entienden a las conexiones matemáticas como un proceso en el que una persona establece una relación verdadera (matemáticamente consistente) entre dos o más ideas, conceptos, teoremas, procedimientos, significados, etc., entre sí, con la vida real o con otras disciplinas. Estas emergen, según los autores, en el momento en que los estudiantes resuelven tareas específicas y se pueden identificar en sus producciones escritas y en los argumentos orales o gestuales que desarrollen. Esta es la definición que se asume en la investigación.

\subsection{MARCO DE REFERENCIA PARA ESTUDIAR CONEXIONES MATEMÁtICAS}

La literatura en Matemática Educativa muestra que no hay un enfoque único para estudiar conexiones matemáticas. Por ejemplo, encontramos investigaciones como las de Businskas (2008) y Eli et al. (2013) que, a partir de sus datos, construyen algunas categorías de conexiones matemáticas que los profesores de matemáticas establecen. Sin embargo, es posible que los estudiantes utilicen otras tipologías no identificadas en esas investigaciones. Por esta razón, nosotros utilizamos las tipologías propuestas por García (2018) y García-García (2019) como un marco de referencia para estudiar conexiones matemáticas (ver figura 1) porque fueron construidas a partir de datos colectados con estudiantes (aunque lo hacen para el concepto de derivada e integral). Creemos que el uso de este marco puede extenderse a cualquier otro concepto involucrado en las tareas matemáticas que resuelvan los estudiantes. En este sentido, consideramos que esta investigación podría validar esta conjetura, este es, entre otros, el aporte de este estudio.

El marco de referencia considerado se estructura en las siguientes tipologías de conexiones matemáticas, las mismas que son consideradas en esta investigación.

- Procedimental. Aparece cuando alguien utiliza reglas, algoritmos o fórmulas establecidas dentro de un registro semiótico para resolver tareas matemáticas. Tales conexiones son de la forma $A$ es un procedimiento utilizado cuando se trabaja con el objeto B. En este sentido, puede incluir todo aquel medio que sirva para llegar a un resultado, por ejemplo, usar una gráfica como procedimiento. 
- Representaciones diferentes. Esta emerge cuando un mismo concepto matemático es expresado en registros semióticos diferentes, por ejemplo: lenguaje natural-gráfico, algebraico-gráfico, numérico-tabular, etc. O bien, expresado en dos formas distintas dentro de un mismo registro.

- Característica. Se manifiesta cuando un estudiante es capaz de identificar un atributo invariante o rasgo de un concepto matemático que lo puede distinguir de otro. $\mathrm{O}$ bien, para identificar elementos comunes entre dos o más conceptos, procedimientos o representaciones distintas.

- Reversibilidad. Es referida a las relaciones bidireccionales entre dos operadores matemáticos, es decir, se puede partir de un concepto A para llegar a un concepto $\mathrm{B}$ e invertir el proceso partiendo de B para regresar al A. Esto implica que los estudiantes pueden partir de un punto final y seguir el curso de un razonamiento hasta llegar a un punto inicial y viceversa.

- Significado. Se identifica cuando los estudiantes le atribuyen un sentido a un concepto matemático en tanto lo que para ellos es (que lo hace diferente de otro) y lo que representa, puede incluir la definición que ellos han construido para estos conceptos. Asimismo, incluye su contexto de uso.

- Parte-todo. Se exterioriza en el momento en que los estudiantes establecen relaciones lógicas entre conceptos matemáticos, bien sean de generalización (entre casos generales y particulares) o de inclusión (un concepto matemático contenido en otro).

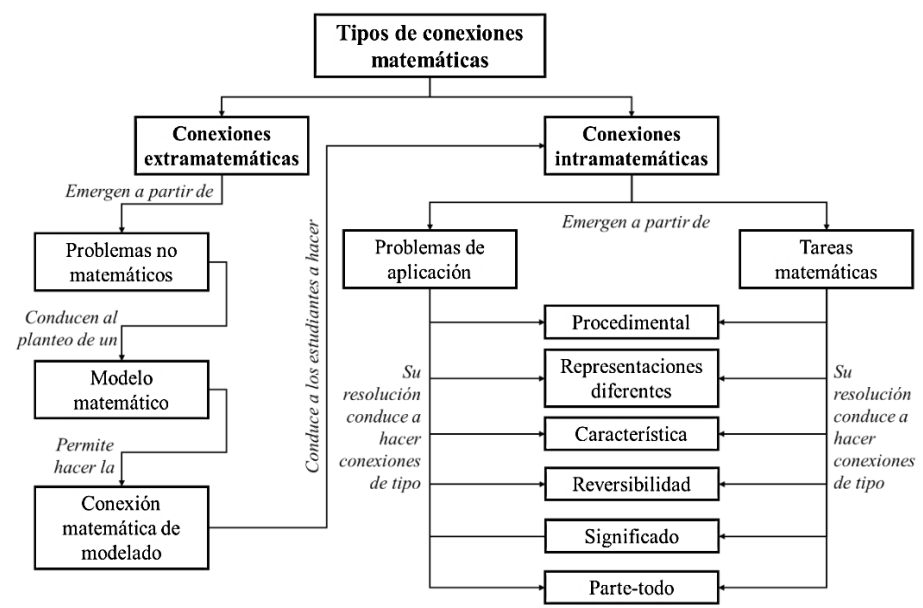

Figura 1. Tipologías de conexiones matemáticas (Adaptada de García, 2018, p. 166). 


\section{METODOLOGÍA}

La presente investigación es cualitativa, específicamente es un estudio de casos. Este método es una forma de hacer investigación a profundidad de una unidad social (ya sea una persona, una familia, una institución, un grupo cultural, etc.), lo que implica la observación cuidadosa y el análisis completo de un número limitado de eventos o condiciones. Su objetivo es localizar los factores que explican los patrones de comportamiento de la unidad dada como una totalidad integrada (Kothari, 2004). De manera general, en este trabajo seguimos la siguiente ruta metodológica: diseño (selección del caso y diseño del protocolo), preparación, colecta de datos, análisis y conclusión.

Como método para la colecta de datos se empleó la Entrevista Basada en Tareas, ya que permite entrevistar al sujeto justo mientras resuelve una tarea matemática, posibilitando la recolección de información escrita y verbal (Goldin, 2000).

\subsection{CONTEXTO DE LA INVESTIGACIÓN Y SELECCIÓN DE LOS PARTICIPANTES}

La investigación se llevó a cabo en una universidad pública de Cali, Colombia, en una Facultad de Ingeniería -donde estudian las funciones exponencial y logarítmica en primer semestre en la asignatura de Cálculo I- y en una Facultad de Educación y Pedagogía -donde las funciones en estudio las trabajan en segundo semestre en la asignatura de Iniciación al Álgebra-. Los programas académicos en esta institución tienen una duración de cinco años (equivalente a 10 semestres en México).

Los participantes fueron siete estudiantes universitarios (dos hombres y cinco mujeres) de diferentes semestres, cuatro de ellos, de la licenciatura en Educación Básica con Énfasis en Matemáticas y, los otros tres, de Ingeniería. Para seleccionar a los estudiantes se consideraron los siguientes criterios: (1) que hubiesen cursado y aprobado el curso de Iniciación al Álgebra, para el caso de los de licenciatura y, Cálculo I, para el caso de los ingenieros y, (2) que estuvieran dispuestos a colaborar de manera voluntaria en esta investigación. Los estudiantes no cursaban los mismos semestres ni la misma carrera. En adelante, llamaremos a estos casos como: E1, E2, E3, E4, E5, E6 y E7.

Los estudiantes de licenciatura son E1, E2, E3 y E4. E1 tenía 22 años y se encontraba cursando octavo semestre; E2 tenía 20 años y cursaba sexto 
semestre; E3 tenía 20 años y cursaba cuarto semestre y E4 tenía 23 años y cursaba décimo semestre. Los estudiantes de ingeniería son E5 de 19 años que cursaba sexto semestre de Ingeniería Industrial; E6 de 30 años que cursaba décimo semestre de Ingeniería Eléctrica y E7 de 21 años que cursaba noveno semestre de Ingeniería Civil.

\subsection{INSTRUMENTO}

Para la construcción y diseño de las Entrevistas Basadas en Tareas, Goldin (2000) establece diez principios, de los cuales se consideraron los siguientes: se seleccionaron tareas que fueran asequibles a los sujetos que las van a desarrollar; tareas que incluyen estructuras ricas en representaciones; se fomentó la libre resolución de problemas; se decidió previamente qué grabar tanto como fue posible; se prepararon entrevistas y un test piloto y se previeron nuevas posibilidades o imprevistos.

Considerando estos criterios se analizaron las tareas sobre las funciones exponencial y logarítmica que plantean los libros de texto: Precálculo de Stewart et al. (2012) y Cálculo de Larson y Edwards (2010), además del documento la teoría de los campos conceptuales y la conceptualización en el aula de matemática y física de Otero et al. (2014). Se buscó que esas tareas fueran asequibles y permitieran una variedad de reflexiones y argumentos de los estudiantes al momento de resolverlas. Una vez diseñado el instrumento inicial con seis tareas, fue validado mediante dos procedimientos: una validación de expertos -fue valorado por tres investigadores que han trabajado en la línea de conexiones matemáticas- y una aplicación piloto con estudiantes de bachillerato y de nivel superior. Hecha la validación, se rediseñaron las tareas quedando conformado el instrumento final por cuatro tareas (tabla 1 y figura 2).

Mientras los estudiantes resolvían cada tarea, se plantearon preguntas auxiliares para indagar o profundizar en el razonamiento que ponían en juego. Por ejemplo, en la tarea 3.d. se plantearon dos preguntas que fueron consideradas en el protocolo utilizado por el entrevistador: ¿Existe alguna relación entre las dos funciones encontradas? ¿Cuál? Asimismo, se recopiló información sobre datos personales de los participantes. 
Tabla 1. Tareas planteadas a los estudiantes

1. Completa la siguiente tabla y explica la estrategia usada.

\begin{tabular}{|c|c|c|c|c|c|c|c|c|c|c|c|c|}
\hline-3 & -2 & & 0 & 1 & 2 & 3 & & 5 & & 7 & & 9 \\
\hline $1 / 8$ & $1 / 4$ & & 1 & 2 & 4 & 8 & 16 & 32 & & & & \\
\hline
\end{tabular}

a. Encuentra una expresión matemática que relacione el término de la segunda fila con el término de la primera fila.

b. ¿Qué términos de la primera fila se corresponde con 1024 y $\frac{1}{6384}$ de la segunda?

2. Una enfermedad infecciosa comienza a esparcirse en un grupo de 10,000 estudiantes en una universidad. Después de $t$ días, el número de personas infectadas se modela mediante la función:

$$
v(t)=\frac{10000}{5+1245 e^{-k t}}
$$

Donde $t$ significa tiempo medido en días y $k$ una constante

a. ¿Cuántas personas infectadas hay inicialmente?

b. Si después de 2 días hay 54 personas infectadas (Sugerencia: con este dato puedes encontrar el valor de $k$ ). Considerando el valor de $k$ que encontraste: ¿Cuántas personas estarán infectadas en 5 días?

c. Considerando $k=1$, la gráfica que modela el número de personas infectadas es la que se muestra enseguida. Observa la gráfica (ver figura 2) y responde las preguntas que se plantean.

- ¿En cuánto tiempo habrá 1000 personas infectadas?

- ¿Cuál es el dominio de la función v(t)? ¿Cuál es el dominio que tiene sentido en la situación planteada?

- ¿Durante qué días la infección se propaga lentamente?

- ¿Durante qué días la infección se propaga con mayor rapidez?

3. La relación de Ehrenberg $\ln w=\ln 2.4+(1.84) h$ es una fórmula empírica que relaciona la altura $h$ (en metros) con el peso promedio $w$ (en kilogramos) para niños entre 5 y 13 años.

a. Si una niña de 8 años pesa $28.8 \mathrm{~kg}$ ¿Cuál es su estatura promedio?

b. ¿Cuál es el peso promedio de un niño de 10 años cuya altura es de 1.5 metros?

c. ¿Cuál es la función que permite conocer el peso promedio para cualquier niño cuya edad varía entre 5 y 13 años? Grafique la función.

d. ¿Cuál es la función que permite conocer la estatura promedio para cualquier niño cuya edad varía entre 5 y 13 años? Grafique la función.

4. Define qué es función exponencial y función logarítmica. ¿Existe alguna relación entre ellas? ¿Cuál? 


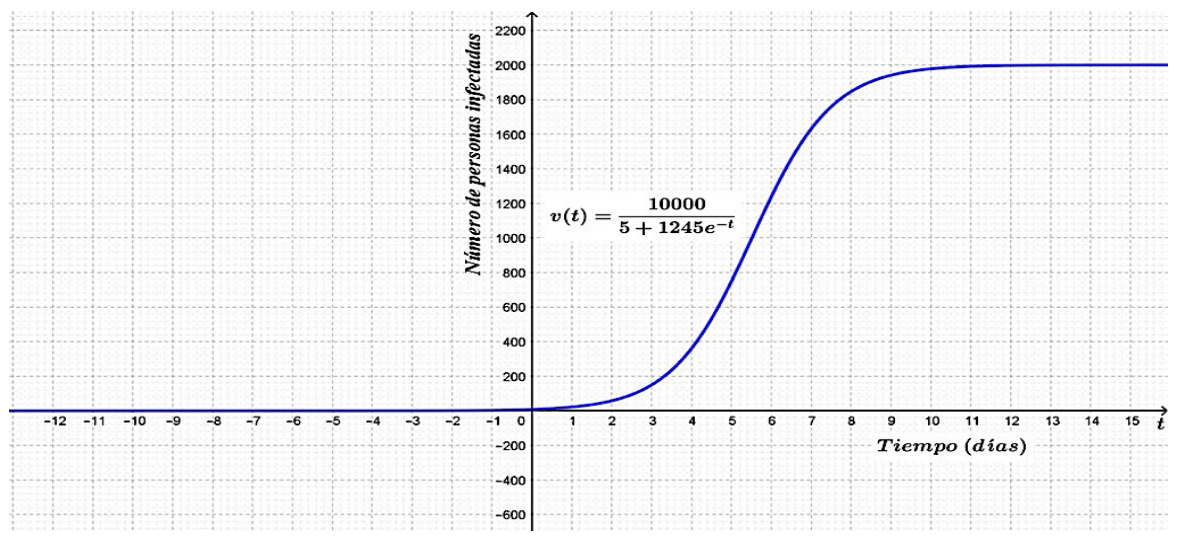

Figura 2. Gráfica de la tarea 2.

\subsection{ANÁLISIS DE LOS DATOS}

Para el análisis de los datos se empleó el Análisis Temático, el cual permite identificar y examinar patrones en datos cualitativos (Clarke y Braun, 2013). Su objetivo es identificar patrones de significados (temas) a través de un conjunto de datos proporcionados por las respuestas a la pregunta de investigación planteada. Según Braun y Clarke (2006), "un tema capta algo importante acerca de los datos en relación con la pregunta de investigación y representa cierto nivel de patrón respuesta o significado dentro del conjunto de datos" (p. 86, traducción nuestra). Elegimos usar este método por las siguientes razones: (1) es un método flexible que permite trabajar con pocos o muchos datos, (2) permite un análisis basado en datos o teoría -en nuestro caso, fue basado en teoría porque usamos previamente un marco de referencia para estudiar conexiones matemáticas- y (3) permite responder una variedad de preguntas de investigación.

Este método se estructura en seis fases (Braun y Clarke, 2006, 2012):

Fase 1. Familiarización con los datos.

Implica conocer a detalle las producciones (verbales, escritas o gestuales) de los estudiantes mediante la lectura general de las narrativas en repetidas ocasiones. Mientras se hizo esto, tomamos notas de algunas observaciones importantes asociadas a las primeras conexiones matemáticas que identificamos. 
Fase 2. Generación de códigos iniciales.

En ella se organizó la información en grupos de un mismo significado. Para ello se codificó la mayor cantidad posible de patrones respuesta y se incorporó en cada código la información suficiente para no olvidar la perspectiva contextual. Los códigos iniciales se obtuvieron a través de las lecturas realizadas en la fase 1 y de una codificación teórica prevista sobre conexiones matemáticas. Para ello, en las narrativas se identificaron proposiciones donde los estudiantes relacionaran o vincularan dos o más ideas matemáticas. Por ejemplo, E4 argumentó "la relación (matemática) como tal entre las dos funciones (función exponencial y logarítmica) es que son inversas" cuando se le cuestionó sobre las funciones exponenciales y logarítmicas, lo que nos permitió construir el código: las funciones exponencial y logarítmica son funciones inversas. Esta codificación se fue refinando conforme avanzaba el proceso hasta analizar todas las narrativas de los estudiantes entrevistados.

Fase 3. Búsqueda de temas y subtemas.

En esta fase se revisaron los datos codificados para identificar áreas de similitud, se agruparon aquellos que compartían algún rasgo de tal manera que se formaron familias de códigos y se describió la característica particular de cada una. Teniendo en cuenta esto, se asignó un tema o subtema a cada agrupación de códigos que respondiera la pregunta de investigación. Como convención, en este trabajo, cada tema y subtema corresponden a las conexiones matemáticas específicas. Se usaron como temas las tipologías de conexiones matemáticas consideradas en el marco teórico y los subtemas se construyeron a partir de los datos y se organizaron en torno a cada tema.

Fase 4. Revisión de temas y subtemas.

Consiste en una recodificación y descubrimiento de nuevos temas. Para ello se realizó una relectura de los temas y subtemas establecidos. En esta revisión consideramos que estos respondieran a la pregunta de investigación planteada y mantuvieran coherencia interna entre los datos. De esta manera, pudimos crear y modificar los temas y subtemas. Por ejemplo, el subtema la tabla de valores registra los valores de una función exponencial de base 2 fue modificado por Las parejas ordenadas ...; $(-1,1 / 2) ;(0,1) ;(1,2) ; . .$. representan la función exponencial de base 2 .

Fase 5. Definición y denominación de temas.

Requiere que el investigador realice y escriba detalladamente el análisis de cada tema, identificando la esencia de cada uno, a fin de construirle un nombre 
conciso e informativo (Braun y Clarke, 2012). En nuestro caso definimos los temas identificados y explicamos los subtemas construidos con los datos.

Fase 6. Redacción del informe.

Consiste en una escritura sustentada en la comprensión e interpretación de la información recogida. Para ello se debe tener un conjunto de temas completamente definidos y analizados para proceder a la redacción del escrito, lo cual implica unir la narrativa analítica y los extractos de datos, a fin de contarle al lector una historia coherente y persuasiva sobre los datos y contextualizarla en la literatura existente.

\section{RESULTADOS}

El análisis de las producciones escritas y verbales de los siete estudiantes indican que estos utilizan en un nivel distinto las conexiones matemáticas (ver tabla 2). Estas se corresponden con las previstas en el marco conceptual -los datos no permitieron construir alguna otra tipología de conexión matemática- por lo que podemos argumentar que este es pertinente y válido cuando se exploran las conexiones matemáticas que los estudiantes establecen al resolver tareas matemáticas que involucran distintos conceptos matemáticos. A continuación, presentamos los resultados de acuerdo con cada tipología de conexión matemática, mostrando algunos ejemplos.

Tabla 2. Conexiones matemáticas esperadas y obtenidas en las tareas propuestas

\begin{tabular}{|c|c|c|}
\hline Tarea & $\begin{array}{l}\text { Conexiones matemáticas } \\
\text { esperadas }\end{array}$ & Estudiantes que la evidenciaron \\
\hline \multirow{6}{*}{ Tarea 1} & Característica & \multirow{5}{*}{ Todos } \\
\hline & Procedimental & \\
\hline & Significado & \\
\hline & Reversibilidad & \\
\hline & Parte-todo & \\
\hline & Representaciones diferentes & Rt-Rs (Todos) y Rt-Rs-Rg (Solo E1) \\
\hline
\end{tabular}




\begin{tabular}{|c|c|c|}
\hline \multirow{5}{*}{ Tarea 2} & Característica & \multirow{4}{*}{ Todos } \\
\hline & Procedimental & \\
\hline & Reversibilidad & \\
\hline & Parte-todo & \\
\hline & Representaciones diferentes & Rs-Rg (Solo E1, E4 E5) \\
\hline \multirow{5}{*}{ Tarea 3} & Característica & \multirow{2}{*}{ Todos } \\
\hline & Procedimental & \\
\hline & Reversibilidad & Rs (Todos), Rg (Solo E4), entre dominios (E1, E2, E4, E6) \\
\hline & Representaciones diferentes & Rs-Rg (Todos menos E7), Rs-Rg-RIn (Solo E6). \\
\hline & Parte-todo & Todos a excepción de E7 \\
\hline \multirow{3}{*}{ Tarea 4} & Característica & \multirow{2}{*}{ Todos } \\
\hline & Reversibilidad & \\
\hline & Significado & Todos a excepción de E7 \\
\hline
\end{tabular}

Nota: en la tabla las siglas Rs significa registro simbólico, Rt registro tabular, Rg registro gráfico y RIn registro lenguaje natural.

\subsection{CONEXIÓN MATEMÁTICA CARACTERÍSTICA}

Se esperaba que esta conexión fuera establecida por los estudiantes en las cuatro tareas, lo cual ocurrió. Fue una de las conexiones que todos los casos evidenciaron completamente en sus respuestas, inclinándose más por lo que caracteriza a la función exponencial. Los subtemas más representativos y que se evidenciaron en mayor medida en las producciones de los estudiantes se presentan en la tabla 3. 
Tabla 3. Resultados relacionados con el tema conexión matemática característica

\begin{tabular}{|c|c|}
\hline Subtemas & Estudiantes \\
\hline $\begin{array}{l}\text { Las parejas ordenadas }(-1,1 / 2) ;(0,1) ;(1,2) ; \ldots \text { representan a la fun- } \\
\text { ción exponencial de base } 2 \text {. }\end{array}$ & E1, E2 y E7 \\
\hline $\begin{array}{l}\text { El dominio de } f(x)=2^{x} \text { es el intervalo }(0, \infty) \text {. } \\
\text { La base de una función exponencial debe ser mayor a cero y diferente } \\
\text { de } 1 . \\
\text { La función exponencial tiene la variable independiente en el exponente. }\end{array}$ & E1 y E7 \\
\hline $\begin{array}{l}\text { Para la función } 2^{\mathrm{x}} \text {, la sucesión..., }-2,-1,0,1,2,3, \ldots, \text { son algunos valores } \\
\text { que toma la variable } x \text { y la sucesión } 1 / 2,1,2,4,8, \ldots \text {, son algunos valo- } \\
\text { res de su conjunto imagen. }\end{array}$ & E1 y E2 \\
\hline La función logaritmo natural tiene como base al número $e$. & $\mathrm{E} 1, \mathrm{E} 2, \mathrm{E} 4, \mathrm{E} 5$ y E7 \\
\hline $\begin{array}{l}\text { El dominio de la función } v(t)=\frac{\overline{10000}}{5+1245 e^{-k t}} \text { es el conjunto de los núme- } \\
\text { ros reales y el que tiene sentido en la situación es }[0, \infty) \text {. }\end{array}$ & E2, E3 y E4 E6 \\
\hline $\begin{array}{l}\text { El argumento de un logaritmo debe ser mayor a cero para que tenga } \\
\text { una solución real. }\end{array}$ & E2 y E4 \\
\hline $\begin{array}{l}\text { La diferencia de dos logaritmos es igual al logaritmo del cociente de sus } \\
\text { argumentos }\left(\log a-\log b=\log \frac{a}{b}\right) \text {. }\end{array}$ & E1, E2, E5, E6 \\
\hline $\begin{array}{l}\text { En el producto de dos números con la misma base, los exponentes se } \\
\text { suman }\left(a^{\mathrm{x}} a^{\mathrm{y}}=a^{\mathrm{x}+\mathrm{y}}\right) \text {. }\end{array}$ & E5, E6 \\
\hline La función $f(x)=2^{\mathrm{x}}$ es una función exponencial. & E4 y E6 \\
\hline
\end{tabular}

Un ejemplo particular es el caso de E1, quien evidenció la conexión característica en la resolución de las tareas 1, 2 y 3 porque logró inferir a partir de sus características el comportamiento exponencial de los datos. Además, caracterizó los valores que puede tomar la base de una función exponencial y logarítmica, la relación entre las variables implicadas, las intersecciones de las gráficas con los ejes, el dominio y el rango de las dos funciones involucradas, además de indicar algunas aplicaciones de la función exponencial.

En la tarea 1 (ver extracto de la entrevista E1), E1 describió la relación de los términos de la tabla y esbozó dos características de la función exponencial: la variable independiente está en el exponente y la base de una función exponencial debe ser mayor a cero y diferente de 1 (tabla 3). E1 manifestó esta última 
característica en la tarea 4 para la función logarítmica y, describió cuál es el dominio y rango de esta función en general, tal como se muestra en la figura 3. No obstante, estas dos funciones difieren en que en la función exponencial la variable independiente está en el exponente y en la logarítmica es parte del argumento.

Entrevistador: Lo que dijiste ahora ¿Cuál es la relación que encontraste lentre los datos de las dos filas de la tarea 1]?

E1: $\quad$ Los de arriba corresponden al exponente y los de abajo al resultado de la función exponencial $2^{x}$

Entrevistador: ¿Por qué es función exponencial?

E1: $\quad$ Porque lo que esté elevado es la variable, o sea $x$, que puede tomar cualquier valor, y que debe ser mayor a cero y diferente de 1; es la base.

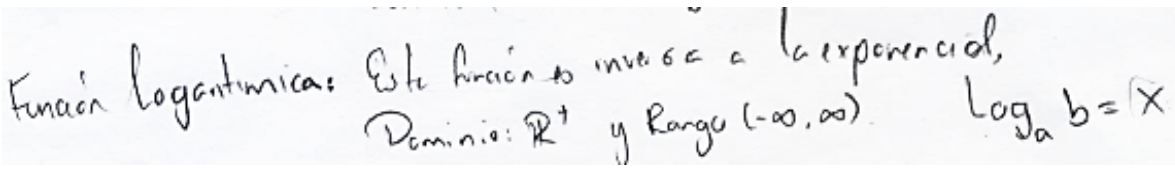

Figura 3. Característica de la función logarítmica según E1.

En lo que respecta a la característica la función logaritmo natural tiene como base al número e, tenemos que E5 la evidenció cuando explicó el porqué de un procedimiento que implicaba despejar una variable en una ecuación exponencial $\left(1245 e^{-t k}=\frac{10000}{54}-54\right)$, como se muestra en el siguiente extracto:

E5:

Hay que despejar $k$ [en $\left.\boldsymbol{v}(\boldsymbol{t})=\frac{\mathbf{1 0 0 0 0}}{\mathbf{5 + 1 2 4 5 \boldsymbol { e } ^ { - t \boldsymbol { k } }}}\right]$, $t$ vendría siendo 2 y $v(t)=54$ Pasé a multiplicar el denominador y lo distribuí de una vez.

Entrevistador: ¿¿Qué propiedad usaste?

E5: Multipliqué a ambos lados por este número que está en el denominador entonces el de abajo ya se cancela o se simplifica.

Aplico logaritmo natural a ambos lados.

Entrevistador: ¿Qué pasa con el logaritmo natural y la $e$ ?

E5: $\quad$ Es que el logaritmo natural tiene base $e$, entonces el exponente se baja y el logaritmo natural de $e$ es 1 . El resultado es 0.98 y ese es el valor de $k$. Pero quiero corroborar reemplazando el 2 en $t$ y el 0.98 en $k$ y si me da 54 es porque es correcto [el resultado]. 


\subsection{CONEXIÓN MATEMÁtICA PROCEDIMENTAL}

En las tareas planteadas a los estudiantes se había previsto que la conexión procedimental fuera establecida por los siete casos en las tres primeras tareas, tal como ocurrió. Fue empleada, principalmente, en el registro algebraico, aunque algunos de los estudiantes usaron también el registro gráfico como procedimiento. Esta conexión apareció en los procesos que emplearon para resolver ecuaciones exponenciales y logarítmicas en el que usaron fórmulas, propiedades de los números reales y de las funciones en estudio. En la tabla 4 se muestran los subtemas más representativos de la conexión procedimental.

Tabla 4. Resultados relacionados con el tema conexión matemática procedimental

\begin{tabular}{lc}
\hline \hline \multicolumn{1}{c}{ Subtemas } & Caso de estudio \\
\hline $\begin{array}{l}\text { Analizando la gráfica de } v(t)=\frac{10000}{5+1245 e^{-k t}} \text { se encuentra el número de } \\
\text { personas infectadas y se puede ver cómo es la propagación de la enfer- }\end{array}$ & \\
medad. & E3, E4 y E6 \\
\hline $\begin{array}{l}\text { Para despejar } w \text { en } \ln w=\ln 2.4+(1.84)(1.5) \text { se aplican los axio- } \\
\text { mas de campo de los reales y el número de Euler. }\end{array}$ & E3, E5, E6 y E7 \\
\hline
\end{tabular}

Para resolver una ecuación exponencial se usa el logaritmo natural $(\ln )$ E1, E2 y E3 para despejar el exponente.

Para resolver una ecuación logarítmica se usa el número de Euler para E1, E2, E4, E6 y E7 quitar el logaritmo natural $(l n)$ y los axiomas de campo para despejar la incógnita.

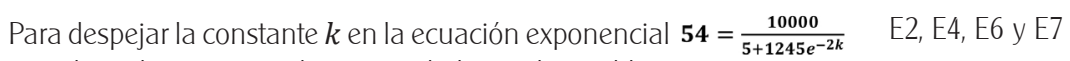
se aplican los axiomas de campo de los reales y el logaritmo.

De manera particular, respecto al subtema para despejar $w$ en $\ln w=\ln$ $2.4+(1.84)(1.5)$ se aplican los axiomas de campo de los reales y el número de Euler, se ejemplifica lo realizado por E2 en la tarea 3.a. E2 reemplazó el valor de la altura en la expresión dada, obteniendo así una ecuación logarítmica en la que para despejar $w$ hizo uso del proceso inverso a la logaritmación, elevando las expresiones al número $e$ (número de Euler, base del logaritmo natural) y empleando la propiedad $e^{\ln x}=x$ para encontrar un determinado valor (ver figura 4). 


\section{$3 a \ln \omega=\ln 2.4+(1.84) 1.5 \Rightarrow \ln \omega=0.87+2.76$ $\Rightarrow \ln \omega=3.63 \Rightarrow e^{\ln \omega}=e^{3.63} \Rightarrow \omega=37.71$ El niño pesa $37.21 \mathrm{~kg}$ con la altura de 1.5}

Figura 4. Conexión procedimental hecha por E2.

Respecto al subtema analizando la gráfica de $v(t)=\frac{10000}{5+1245 e^{-k t}}$ se encuentra el número de personas infectadas y se puede ver cómo es la propagación de la enfermedad, E3 lo evidenció al explicar cómo encontró el tiempo en el que hay 1000 personas infectadas y cuando manifestó que la propagación se puede visualizar analizando el eje y (ver extracto de E3).

Entrevistador: Pero dime exactamente cien cuántos días?

E3: $\quad$ En 6 días

Entrevistador: ¿'Por qué?

E3: $\quad$ Porque a mí aquí en la gráfica me da como la mitad, pero en 6 días habrá 1200 infectados, a mí me da los 1000 aquí en la mitad (toca entre el 5 y el 6), en 5 días y medio.

Entrevistador: ¿¿Usted considera que se infectarán a lo máximo 2000?

E3: $\quad$ De acuerdo con la gráfica solo 2000 porque veo que es constante.

Entrevistador: Para ver los días en que se propaga más lento, ¿̇ué estas mirando?

E3: $\quad$ El eje y para ver el cambio, la cantidad de personas que va aumentando, porque de 4 a 5 días la infección se ha propagado a muchas personas, en cambio de 0 a 3 sigue estando en la misma zona aproximadamente, entonces digo que es en esos días.

\subsection{CONEXIÓN MATEMÁtICA DE SIGNIFICADO}

Esta se evidenció en las producciones de seis de los siete casos, ya que E7 no logró, en la tarea 4, dar una definición consistente de las funciones exponencial y logarítmica. En la tabla 5 se muestran los subtemas más representativos de esta. 
Tabla 5. Resultados relacionados con el tema conexión matemática de significado

\begin{tabular}{|c|c|}
\hline Subtemas & Caso de estudio \\
\hline $\begin{array}{l}\text { Una función exponencial es aquella en la que un número real está } \\
\text { elevado a la variable independiente. }\end{array}$ & $\mathrm{E} 1, \mathrm{E} 2, \mathrm{E} 4, \mathrm{E} 5$ \\
\hline $\begin{array}{l}\text { Una función logarítmica es aquella donde la variable independiente es } \\
\text { parte del argumento. }\end{array}$ & $\mathrm{E} 1, \mathrm{E} 2, \mathrm{E} 3, \mathrm{E} 4, \mathrm{E} 6$. \\
\hline Todo número diferente de cero elevado a la potencia cero es uno. & $\mathrm{E} 1, \mathrm{E} 2, \mathrm{E} 4, \mathrm{E} 7$ \\
\hline Una función es una relación biunívoca entre dos conjuntos. & $\mathrm{E} 1, \mathrm{E} 3, \mathrm{E} 5$ \\
\hline $\begin{array}{l}y=2^{x} \text { es una función exponencial porque tiene la variable indepen- } \\
\text { diente en el exponente. }\end{array}$ & E2 \\
\hline
\end{tabular}

La conexión de significado apareció en distintos momentos mientras los estudiantes resolvieron las tareas propuestas. Por ejemplo, E1 evidenció el subtema una función exponencial es aquella en la que un número real está elevado a la variable independiente cuando enunció lo que entiende por función exponencial y, además, estableció las condiciones de la base, describió de manera general el dominio y el rango de la función (ver figura 5).

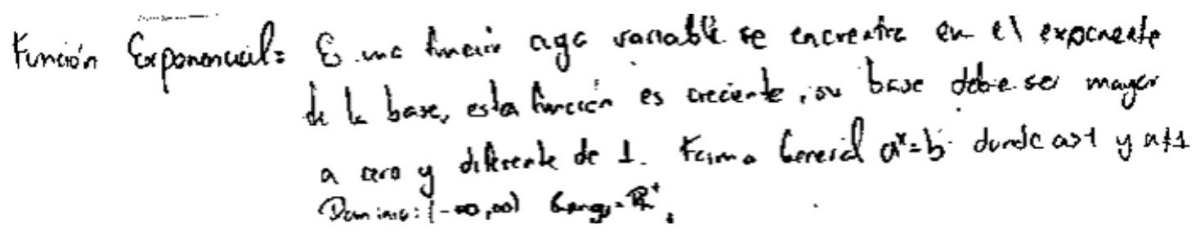

Figura 5. Definición de función exponencial por E1.

E4 evidenció el subtema una función logarítmica es aquella donde la variable independiente es parte del argumento cuando enunció lo que entiende por función logarítmica (ver extracto E4).

Entrevistador: ¿Qué sería una función logarítmica?

E4: $\quad$ Que la variación esté dentro de la función logaritmo, la variable esté encerrada dentro de un logaritmo.

De manera general, la mayoría de los casos evidenciaron una definición de las funciones exponencial y logarítmica acorde a la definición matemática aceptada. 


\subsection{CONEXIÓN MATEMÁTICA DE REVERSIBILIDAD}

La conexión matemática de reversibilidad fue establecida por todos los casos, en tres de las cuatro tareas. Principalmente, en el momento de resolver ecuaciones exponenciales y logarítmicas ya que, consideraron necesario el uso del número de Euler para resolver una ecuación logarítmica y el logaritmo para despejar una ecuación exponencial. Los subtemas más representativos que se construyeron a partir de los datos se describen en la tabla 6 .

Tabla 6. Resultados relacionados con el tema conexión matemática de reversibilidad

\begin{tabular}{|c|c|}
\hline Subtemas & Estudiantes \\
\hline Las funciones exponencial y logarítmica son funciones inversas. & E1, E2, E3, E4, E5, E6, E7 \\
\hline $\begin{array}{l}\text { Las funciones } \boldsymbol{h}=\frac{(\ln \boldsymbol{w}-\ln \mathbf{2 . 4})}{\mathbf{1 . 8 4}} \text { y } \boldsymbol{w}=\boldsymbol{e}^{\ln \mathbf{2 . 4 + 1 8 4 (}(\boldsymbol{h})} \text { son inversas y } \\
\text { por lo tanto el dominio de } w \text { es el rango de } h \text { y el rango de } w \text { es } \\
\text { el dominio de } h .\end{array}$ & E1, E2, E4, E6 \\
\hline $\begin{array}{l}\text { El logaritmo natural se usa para despejar el exponente en una } \\
\text { ecuación exponencial. }\end{array}$ & E1, E2, E3, E4, E5, E6, E7 \\
\hline $\begin{array}{l}\text { El número de Euler se usa para despejar, en una ecuación loga- } \\
\text { rítmica, la variable que se encuentra en el argumento del logarit- } \\
\text { mo natural. }\end{array}$ & E1, E2, E3, E4, E5, E6, E7 \\
\hline
\end{tabular}

Las gráficas de las funciones exponencial y logarítmica se reflejan E4 mediante un eje de simetría.

Un ejemplo particular del subtema el logaritmo natural se usa para despejar el exponente en una ecuación exponencial y el Euler para despejar la variable que se encuentre en el argumento del logaritmo natural fue evidenciado por E7 en la pregunta 3. Él afirmó que en una ecuación exponencial para despejar el exponente (variable independiente) se debe hacer uso del logaritmo dado que son funciones inversas (ver extracto de E7).

Entrevistador: ¿Siempre que tenga un exponente que quiera bajar uso logaritmo?

E7: $\quad$ Sí, se supone que es la inversa de la exponencial. 
Por su parte, el subtema las funciones exponencial y logarítmica son funciones inversas, se evidenció por E5 cuando al explicar su concepto de función logarítmica la define como la inversa de la exponencial (ver figura 6).

\section{Funcón logaitmica \\ huersa de la funcion exponcual, tiene un comportamiento reciente y para bs reales positivoi con asintota en $D_{-}=x$.}

Figura 6. Definición de función logarítmica por E5.

Esta conexión matemática no se evidenció como se había previsto, porque, a pesar de que todos los estudiantes manifestaron que las funciones exponencial y logarítmica eran inversas, solo algunos reconocían que por ser inversas el dominio y el rango de una de las funciones eran el rango y el dominio de la otra. Entre los estudiantes, solo E4 explicó la propiedad gráfica de dos funciones inversas que relacionaba un eje de simetría.

\subsection{CONEXIÓN MATEMÁTICA DE TIPO REPRESENTACIONES DIFERENTES}

Esta conexión matemática no se evidenció en las respuestas de los estudiantes como se había contemplado, ya que fue la menos usada. La mayoría de los casos solo empleó el registro tabular como medio para graficar. Son pocos los estudiantes que se valen de varios registros por sí solos para responder las tareas, por el contrario, usan las representaciones que se les pide o que se les proporciona. Los subtemas más representativos en esta conexión se muestran en la tabla 7. 
Tabla 7. Resultados relacionados con el tema conexión matemática representaciones diferentes

\begin{tabular}{|c|c|}
\hline Subtemas & Estudiantes \\
\hline $\begin{array}{l}\text { Los puntos en el plano }(-1,1 / 2) ;(0,1) ;(1,2) ; \ldots \text { representan una función } \\
\text { exponencial y su gráfica interseca al eje } \boldsymbol{y} \text { en } 1 \text {. }\end{array}$ & E1 \\
\hline $\begin{array}{l}\text { Realizar una tabla de valores para la función } w=e^{\ln 2.4+1.84(h)} \text { permite } \\
\text { construir su gráfica. }\end{array}$ & E1, E2, E4 \\
\hline 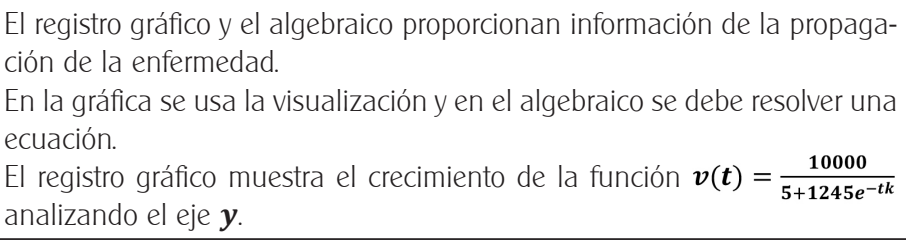 & E2, E4, E5 \\
\hline $\begin{array}{l}\text { La gráfica de la función } w=e^{\ln 2.4+1.84(h)} \text { muestra que es una función } \\
\text { exponencial. }\end{array}$ & \\
\hline
\end{tabular}

Por ejemplo, el subtema la gráfica de la función $w=e^{\ln 2.4+1.84(h)}$ muestra que es una función exponencial se evidenció en la producción de E6 cuando, para determinar qué clase de función representaba la función que modela el peso, hizo una gráfica; para ello, asignó valores a $h$ y encontró los correspondientes valores de $w$ (ver figura 7). Así, al ver la forma de la gráfica afirmó que es una función exponencial, por tanto, hizo uso de tres registros: algebraico, tabular y gráfico en ese mismo orden.

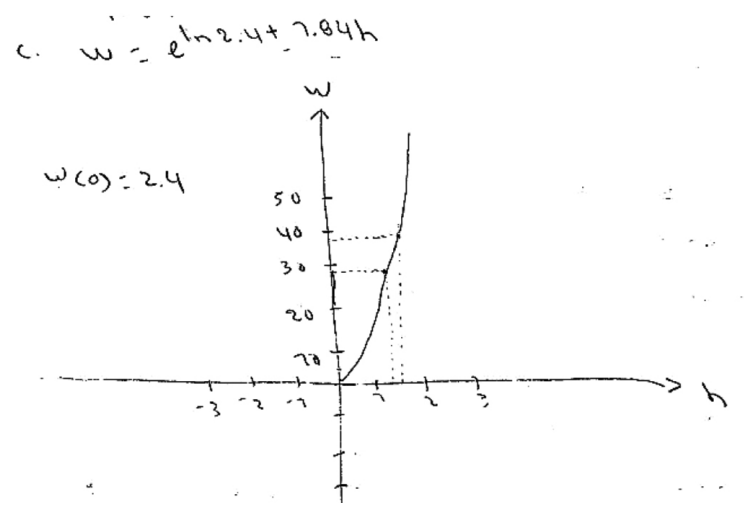

Figura 7. Representaciones diferentes de la función que modela el peso empleadas por E6. 
Por su parte, el subtema el registro gráfico y el algebraico proporcionan información de la propagación de la enfermedad, se identificó en E2 cuando justificó que se puede encontrar la cantidad de personas infectadas mediante la visualización y análisis del registro gráfico o bien resolviendo una ecuación en el registro algebraico, como muestra el siguiente extracto:
E2:
Serían 5 días y 12 horas.
Entrevistador: ¿Esas 12 horas que significan?
E2: $\quad$ Entonces serían 5 días y medio. Pero voy a colocar las horas. ¿Por qué? porque la gráfica me lo indica.
Entrevistador: ¿Habrá otra forma de poder encontrar eso?
E2: $\quad$ Sí, reemplazando el número de personas que nos dan y despejando to parecido a lo que hice cuando despejé.

\subsection{CONEXIÓN MATEMÁTICA PARTE-TODO}

Se identificó en seis de los siete estudiantes, teniendo mayor frecuencia cuando resolvieron las tareas asociadas a la función exponencial. En la tabla 8 se muestran los subtemas más representativos relacionados con esta conexión matemática.

Tabla 8. Resultados relacionados con el tema conexión matemática parte todo

\begin{tabular}{|c|c|}
\hline Subtemas & Estudiantes \\
\hline $\begin{array}{l}\text { Las parejas ordenadas }(-1,1 / 2) ;(0,1) ;(1,2) ; \ldots \text { representan la función ex- } \\
\text { ponencial } 2^{x}\end{array}$ & E1, E2, E3, E4 y E5 \\
\hline $\begin{array}{l}\text { La función exponencial natural es un caso particular de una función exponencial. } \\
\text { La función logaritmo natural es un caso particular de la función logaritmo. }\end{array}$ & E2 \\
\hline $\begin{array}{l}\text { La función } w(h)=2.4 e^{1.8 h} \text { es una función exponencial por su forma gráfica y } \\
\text { algebraicamente es un caso particular de una exponencial } f(x)=a b^{x}\end{array}$ & E2, E5 y E6 \\
\hline La forma general de la función exponencial es $f(x)=a^{x}$ & E4 \\
\hline $\begin{array}{l}\text { La función que modela la estatura }\left(\boldsymbol{h}=\frac{(\boldsymbol{l n} \boldsymbol{w}-\ln \mathbf{2 . 4})}{\mathbf{1 . 8 4}}\right) \text { es una función logarítmica } \\
\text { porque la variable independiente es parte del argumento del logaritmo. }\end{array}$ & E4 y E5 \\
\hline
\end{tabular}


E4, por ejemplo, logró pasar de la expresión que relaciona un caso particular a la forma algebraica general de una función exponencial, lo cual se asocia con el subtema: la forma general de la función exponencial es $f(x)=a^{x}$, como se evidencia en el siguiente extracto:

Entrevistador: ¿¿Qué es una función exponencial?

E4: $\quad$ Es cuando el comportamiento depende de la variación que haya en el exponente.

Entrevistador: ¿¿Sabes cuál es la forma general?

E4:

$$
f(x)=a b^{x} \text {. }
$$

Con mayor frecuencia esta conexión matemática se presentó en su forma de la parte al todo, pero también hubo del todo a la parte cuando los estudiantes debían encontrar casos particulares de las funciones dadas. Por ejemplo, E2 evidenció el subtema: la función logaritmo natural es un caso particular de la función logaritmo (ver extracto E2).

Entrevistador: Respecto a la expresión que colocaste $(\ln x)$ en la función logaritmo cesa es general o particular?

E2: $\quad$ Particular, pero no estoy segura.

Entrevistador: ¿Cuál es la base de esa función?

E2: $\quad 1$.

Entrevistador: La base, ¿el logaritmo natural qué base tiene?

E2: $\quad 10$, o no Euler. La función logaritmo natural es particular. Entonces la general sería (y escribe $f(x)=\log _{b} x$ ).

Lo anterior muestra que E1, aunque inicialmente duda, logra afirmar que la forma general de la función logaritmo tiene una base general y, por tanto, el logaritmo natural es un caso particular de la función logaritmo que tiene como base el número de Euler. 


\section{DISCUSIÓN Y CONCLUSIÓN}

El objetivo de esta investigación fue identificar las conexiones matemáticas que realizan los estudiantes universitarios que cursan carreras afines a la matemática cuando resuelven tareas que involucran a las funciones exponencial y logarítmica. Para lograrlo, se hizo uso de la Entrevista Basada en Tareas a fin de recolectar información escrita y verbal sobre el razonamiento que seguían los estudiantes mientras resolvían las tareas propuestas. Los datos fueron analizados mediante el Análisis Temático basado en teoría, identificándose seis temas que se corresponden con las tipologías de conexiones matemáticas previstas en el marco conceptual.

Los resultados obtenidos permiten plantear algunas reflexiones. Estos indican, entre otras cosas que, los estudiantes parecen tener mayor familiaridad con la función exponencial, ya que la mayoría enuncian con facilidad su definición, características y propiedades, mientras que en algunos casos la función logarítmica solo la definen como la inversa de la exponencial. Por otra parte, los estudiantes suelen trabajar más con el logaritmo natural y, en algunos casos, pensaron que esta era la función general, pero después de realizar una mirada retrospectiva encuentran que es un caso particular, sin embargo, no deja de ser la preferida para solucionar ecuaciones exponenciales.

De manera general, la mayoría de los estudiantes cuando realizaron la tarea 1 , en el momento de encontrar una expresión matemática que relacionara las progresiones dadas, plantearon una relación lineal, como segunda estrategia una cuadrática y, cuando corroboraron que no era ninguna de las dos, analizaron la segunda fila para ver cómo se iban formando tales números. Este resultado es consistente con lo reportado por Sureda y Otero (2013), quienes mencionan que el estudiante cuando se le presentan problemas de variación piensa inmediatamente en una variación lineal.

Los resultados mostraron que, por lo menos, seis estudiantes evidenciaron su capacidad para realizar diferentes conexiones matemáticas (ver tabla 2). Este resultado es consistente con lo que en su conjunto reportan las investigaciones con este enfoque (Dolores y García-García, 2017; Dolores-Flores et al., 2019; Jaijan y Loipha, 2010). Sin embargo, no se evidenciaron tal como se había previsto, pues como se mostró en los resultados y se resume en la tabla 2, las conexiones matemáticas de significado, representaciones diferentes, reversibilidad y parte-todo no fueron establecidas en todas las tareas. Los estudiantes mostraron mayormente persistencia en el uso de fórmulas y procedimientos 
-indicado por una alta frecuencia en el uso de la conexión procedimental- lo cual es consistente con lo reportado por Jaijan y Loipha (2010), Hong y Thomas (2015) y García-García y Dolores-Flores (2018, 2019).

En cuanto a la conexión de representaciones diferentes, tres estudiantes (E2, E4 y E5) trabajaron conectando el registro algebraico y gráfico manifestando que ambos proporcionaban información sobre las situaciones planteadas en el cuestionario y, a partir de la expresión algebraica lograron deducir la gráfica de las funciones en estudio. En este sentido, la falta de establecimiento de esta conexión matemática por los otros cuatro casos representó un obstáculo para completar las tareas que requieren su uso ya que, el empleo combinado de los diferentes registros de representación es necesario para el estudio de las funciones (Sureda y Otero, 2013), permitiendo así el desarrollo de la comprensión de los estudiantes (Barmby et al., 2009). Por lo tanto, se sugiere proponer en el aula actividades en las que se promueva el establecimiento de la conexión de representaciones diferentes empleando los distintos registros de representación para las funciones estudiadas en la presente investigación.

Por otra parte, dados los argumentos esgrimidos por los estudiantes, así como por su utilidad para resolver las tareas propuestas en esta investigación, consideramos que la conexión matemática de reversibilidad fue la conexión central, porque reconoce la relación bidireccional entre las funciones exponencial y logarítmica. Esta conexión matemática fue establecida por los estudiantes, principalmente, en el registro algebraico al resolver ecuaciones exponenciales y logarítmicas. Sin embargo, faltó utilizarla en otros registros como el gráfico e, incluso, emplear la relación de funciones inversas para hallar el domino y el rango. Esto último fue evidencia de la falta de aplicación de las propiedades de funciones inversas entre las funciones objeto de estudio.

Como muestran los resultados, no todas las conexiones matemáticas fueron establecidas por los estudiantes como se tenía previsto. Esto muestra que, incluso en el nivel superior, es necesario promover el uso de las conexiones matemáticas para las funciones exponencial y logarítmica a fin de que los estudiantes tengan una buena comprensión. Si bien la mayoría establecieron las conexiones matemáticas previstas logrando responder consistentemente las tareas, lo ideal es que todos lo hicieran, e incluso debido al nivel académico en el que se encontraban, se esperaba que mostraran un buen dominio de las propiedades de las funciones para resolver las tareas, tuvieran una interpretación sobre la definición de cada una, así como que comprendieran qué significa que ambas sean inversas. 
Estos resultados sugieren que es necesario implementar estrategias en el aula a fin de mitigar estas dificultades y desarrollar una buena comprensión de estas funciones en los estudiantes. Principalmente, porque los ingenieros las usan en varias aplicaciones en su campo y, en el caso de los licenciados porque en su campo de trabajo serán objeto de enseñanza.

En la literatura encontramos, por lo menos, dos enfoques utilizados al estudiar las funciones exponencial y logarítmica de manera articulada: la teoría APOE y el razonamiento covariacional. Este último tiene mayor auge actualmente en la investigación. En el primer enfoque, Weber (2002) encontró que la comprensión de los estudiantes sobre estas funciones es limitada y la mayoría no eran capaces de conectar las leyes exponenciales con las logarítmicas. En el segundo enfoque, Ferrari-Escolá et al. (2016) encontraron que las tareas propuestas promovieron el desarrollo del razonamiento covariacional discreto en los participantes, pero no les permitió pasar al razonamiento covariacional continuo (nivel más alto). Mientras que, Kuper y Carlson (2020) obtuvieron que el refinamiento de la lección trabajada en el artículo podría ser útil para otros investigadores que están diseñando intervenciones para ayudar a los estudiantes a comprender las funciones exponenciales y logarítmicas y sus propiedades.

En estas vías también se presentan barreras para que los estudiantes lleguen a tener una buena comprensión sobre estas funciones. Creemos que una vía para superarlas, es estudiar estas funciones desde el enfoque de las conexiones matemáticas que se pueden establecer con estas, de tal forma que los estudiantes puedan enunciar características de cada función y relacionarlas; reconocer las unidades significantes de las funciones y establecer relaciones entre ellas y los diferentes registros; realizar procedimientos siendo conscientes de las relaciones establecidas y las propiedades utilizadas; emplear los diferentes registros de representación y relacionarlos; generalizar relaciones entre datos (en cualquier registro) y ser capaces de plantear ejemplos particulares de las funciones en general.

Los resultados indican que el marco de referencia elegido (sección 2.2) resultó útil y, por tanto, se puede extender su uso para estudiar las conexiones matemáticas que establecen estudiantes al resolver tareas que involucren cualquier concepto matemático. Por el carácter cualitativo de esta investigación, los resultados obtenidos no son generalizables, en tanto que es un estudio de casos. Asimismo, creemos que el diseño propuesto para colectar datos podría incorporar -en futuras investigaciones- tareas de mayor complejidad que permitan la modelación como lo prevé el marco teórico para estudiar conexiones extramatemáticas. 
También, se podría utilizar otra metodología para colectar datos donde no se tengan previstos algunos resultados ante tareas específicas.

Finalmente, los resultados obtenidos en esta investigación podrían ayudar a plantear tareas que promuevan el establecimiento de conexiones matemáticas. Con esto, se podría contribuir en la mejora de la comprensión de los estudiantes sobre las funciones exponencial y logarítmica en diferentes niveles educativos, y validarlas mediante un experimento de enseñanza. No obstante, aún es necesario seguir explorando las conexiones matemáticas asociadas a estas funciones que son capaces de establecer estudiantes de nivel bachillerato, así como los profesores en servicio, para tener un mejor panorama sobre esta problemática. En ese sentido, en futuras investigaciones se pueden estudiar las conexiones matemáticas asociadas a las funciones exponencial y logarítmica que promueve el profesor o el currículum oficial, por lo que es posible encontrar nuevas tipologías de conexiones. En suma, consideramos que la línea de investigación sobre conexiones matemáticas es un campo poco explorado, pero con creciente interés en la literatura, principalmente internacional.

\section{REFERENCIAS}

Baki, A., Çatı̆ığlu, H., Coştu, S. y Birgin, O. (2009). Conceptions of high school students about mathematics connections to the real-life. Procedia Social and Behavioral Sciences, I (2009), 1407-1407. https://doi:10.1016/j.sbspro.2009.01.247

Barmby, P., Harries, T., Higgins, S. y Suggate, J. (2009). The array representation and primary children's understanding and reasoning in multiplication. Educational Studies in Mathematics, 70(3), 217-241.

Braun, V. y Clarke, V. (2006). Using thematic analysis in psychology. Qualitative Research in Psychology, 3(2), 77-101. http://doi.org/10.1191/1478088706qp063oa

Braun, V. y Clarke, V. (2012). Thematic analysis. En H. Cooper (Ed.), Handbook of Research Methods in Psychology, (57-71). American Psychological Association. https://doi. org/10.1037/13620-004

Bingölbali, E. y Coşkun, M. (2016). A proposed conceptual framework for enhancing the use of making connections skill in mathematics teaching. Education and Science, 41(183), 233-249. http://doi.org/10.15390/EB.2016.4764

Businskas, A. M. (2008). Conversations about connections: How secondary mathematics teachers conceptualize and contend with mathematical connections [Tesis de doctorado no publicada]. Simon Fraser University. 
Brutlag, D. y Maple, C. (1992). Making connections: Beyond the surface. The Mathematics Teacher, 85(3), 230-235. http://www.jstor.org/stable/27967575

Cawley, J. y Foley, T. (2002). Connecting math and science for all students. Teaching Exceptional Children, 34 (4), 14-19. https://doi.org/10.1177/004005990203400402.

Castro, M., González, M., Flores, S., Ramírez, O., Cruz, M. y Fuentes, M. (2017). Registros de representación semiótica del concepto de función exponencial. Parte I. Entreciencias: Diálogos en la Sociedad del Conocimiento, 5(13), 1-12. http://doi.org/10.21933/J. EDSC.2017.13.218.

Chua, B.L. y Wood, E. (2005). Working with logarithms: students' misconceptions and errors. The Mathematics Educator, 82(8), 53-70.

Clarke, V. y Braun, V. (2013). Teaching thematic analysis: Overcoming challenges and developing strategies for effective learning. The Psychologist, 26(2), 120-123.

De Gamboa, G. y Figueiras, L. (2014). Conexiones en el conocimiento matemático del profesor: propuesta de un modelo de análisis. En M. T. González, M. Codes, D. Arnau y T. Ortega (Eds.), Investigación en Educación Matemática XVIII (pp. 337-344). SEIEM.

Dolores C. y García-García, J. (2017). Conexiones intramatemáticas y extramatemáticas que se producen al resolver problemas de cálculo en contexto: un estudio de casos en nivel superior. Bolema: Boletim de Educaçāo Matemática, 31(57), 158-180. http:// doi.org/10.1590/1980-4415v31n57a08

Dolores-Flores, C., Rivera-López, M. I. y García-García, J. (2019). Exploring mathematical connections of pre-university students through tasks involving rates of change. International Journal of Mathematical Education in Science and Technology, 50(3), 369-389. http://doi.org/10.1080/0020739X.2018.1507050

Elia, I., Panaoura, A., Eracleous, A. y Gagatsis, A. (2007). Relations between secondary pupils' conceptions about functions and problem solving in different representations. International Journal of Science and Mathematics Education, 5(3), 533-556. https:// doi.org/10.1007/s10763-006-9054-7

Eli, J. A., Mohr-Schroeder, M. J. y Lee, C. W. (2011). Exploring mathematical connections of prospective middle-grades teachers through card-sorting tasks. Mathematics Education Research Journal, 23, 297-319. https://doi.org/10.1007/s13394-011-0017-0

Eli, J. A., Mohr-Schroeder, M. J. y Lee, C. W. (2013). Mathematical connection and their relationship to mathematics knowledge for teaching geometry. School Science and Mathematics, 113(3), 120-134. https://doi.org/10.1111/ssm.12009

Ellis, A. B., Ozgur, Z., Kulow, T., Dogan, M. F. y Amidon, J. (2016). An exponential growth learning trajectory: students' emerging understanding of exponential growth through covariation. Mathematical Thinking and Learning, 18(3), 151-181. https://doi.org/10.1 080/10986065.2016.1183090 
Ellis, A. B., Ozgur, Z., Kulow, T., Williams, C. y Amidon, J. (2015). Quantifying exponential growth: Three conceptual shifts in creating multiplicative rates of change. The Journal of Mathematical Behavior, 39, 135-155. https://doi.org/10.1016/j.jmathb.2015.06.004

Escobar, N. V. (2014). Elementos históricos para la enseñanza de la función logarítmica en la educación básica. Revista Brasileira de História da Matemática, 14 (29), 83-115.

Evitts, T. (2004). Investigating the mathematical connections that preservice teachers use and develop while solving problems from reform curricula [Tesis de doctorado no publicada]. Pennsylvania State University College of Education.

Ferrari-Escolá, M., Martínez, G. y Méndez-Guevara, M. (2016). "Multiply by adding": Development of logarithmic-exponential covariational reasoning in high school students. The Journal of Mathematical Behavior, 42, 92-108. https://doi.org/10.1016/j.jmathb.2016.03.003

Frykholm, J. A. y Glasson, G. E. (2005). Connecting science and mathematics instruction: Pedagogical context knowledge for teachers. School Science and Mathematics, 105(3), 127-141.

Font, V. (2007). Una perspectiva ontosemiótica sobre cuatro instrumentos de conocimiento que comparten un aire de familia: particular-general, representación, metáfora y contexto. Educación Matemática, 19(2), 95-128.

Gainsburg, J. (2008). Real-world connections in secondary mathematics teaching. Journal of Mathematics Teacher Education, 11(3), 199-219. http://doi.org/10.1007/s10857-0079070-8

García, J. (2018). Conexiones matemáticas y concepciones alternativas a la derivada y a la integral en los estudiantes del preuniversitario [Tesis de doctorado no publicada]. Universidad Autónoma de Guerrero.

García-García, J. y Dolores-Flores, C. (2018). Intra-mathematical connections made by high school students in performing Calculus tasks. International. Journal of Mathematical Education in Science and Technology, 49(2), 227-252. http://doi.org/10.1080/ 0020739X.2017.1355994

García-García, J. y Dolores-Flores, C. (2019). Pre-university students' mathematical connections when sketching the graph of derivative and antiderivative functions. Mathematics Education Research Journal, 1-22. https://doi.org/10.1007/s13394-019-00286-x

García-García, J. y Dolores-Flores, C. (2020). Exploring pre-university students' mathematical connections when solving Calculus application problems. International Journal of Mathematical Education in Science and Technology, 1-25. http://doi.org/10.1080/ 0020739X.2020.1729429.

García-García, J. (2019). Escenarios de exploración de conexiones matemáticas. NúMEROS, 100, 129-133 
Glass, B. (2002). Students connecting mathematical ideas: possibilities in a liberal arts mathematics class. The Journal of Mathematical Behavior, 21, 75-85. https://doi. org/10.1016/S0732-3123(02)00104-9

Godino, J. D., Batanero, C. y Font, V. (2003). Fundamentos de la Enseñanza y el Aprendizaje de las Matemáticas para Maestros. Universidad de Granada.

Goldin, G. A. (2000). A scientific perspective on structured, task-based interviews in mathematics education research. En A. E. Kelly y R. A. Lesh (Eds.), Handbook of Research Design in Mathematics and Science Education (pp. 517-545). Lawrence Erlbaum Associates.

Gruver, J. (2018). A trajectory for developing conceptual understanding of logarithmic relationships. The The Journal of Mathematical Behavior, 50, 1-22. https://doi. org/10.1016/j.jmathb.2017.12.003

Hong, Y. Y. y Thomas, M. O. J. (2015). Graphical construction of a local perspective on differentiation and integration. Mathematics Education Research Journal, 27, 183-200. http://doi.org/10.1007/s13394-014-0135-6

Hurst, C. (2007). Numeracy in action: students connecting mathematical knowledge to a range of contexts. Mathematics: Essential Research, Essential Practice, 1, 440-449

Jaijan, W. y Loipha, S. (2012). Making mathematical connections with transformations using open approach. HRD Journal, 3(1), 91-100.

Ji-Eun L. (2012). Prospective elementary teachers' perceptions of real-life connections reflected in posing and evaluating story problems. Journal of Mathematics Teacher Education, 15(2012), 429-452. http//doi.org/10.1007/s10857-012-9220-5

Koestler, C., Felton, M., Bieda, K. y Otten, S., (2013). Connecting the NCTM Process Standards and the CCSSM Practices. National Council of Teacher of Mathematics United States of America.

Kothari, C.R. (2004). Research methodology: Methods and Techniques. New Age International Publisher.

Kuper, E. y Carlson, M. (2020). Foundational ways of thinking for understanding the idea of logarithm. The Journal of Mathematical Behavior, 57: 100740. http://doi.org/10.1016/j. jmathb.2019.100740

Larson, R., y Edwards, B. (2010). Cálculo 1 de una Variable. Novena edición. McGRAW-HILL/Interamericana Editores, S.A.

Ministerio de Educación Nacional (2006). Estándares Básicos de Competencias en Matemáticas. Ministerio de Educación Nacional.

Mhlolo, M. K. (2012). Mathematical connections of a higher cognitive level: A tool we may use to identify these in practice. African Journal of Research in Mathematics, 
Science and Technology Education, 16(2), 176-191. http://doi.org/10.1080/10288457.2 012.10740738

Moon, K., Brenner, M. E., Jacob, B. y Okamoto, Y. (2013). Prospective secondary mathematics teachers 'understanding and cognitive difficulties in making connections among representations. Mathematical Thinking and Learning, 15(3), 201-227. https://doi.org/10.1080/10986065.2013.794322

National Council of Teachers of Mathematics (1989). Curriculum and Evaluation Standards for School Mathematics. National Council of Teachers of Mathematics

National Council of Teachers of Mathematics (2007). Principios y Normas para la Matemática Escolar. APM.

Noss, R., Healy L., y Hoyles, C. (1997). The construction of mathematical meanings: connecting the visual with symbolic. Educational Studies in Mathematics, 33, 203-233.

Otero, M., Fanaro, M., Sureda, P., Llanos, V. y Arlego, M. (2014). La Teoría de los Campos Conceptuales y la Conceptualización en el Aula de Matemática y Física. Editorial Dunken.

Sureda, P. y Otero, M. (2013). Estudio sobre el proceso de conceptualización de la función exponencial. Educación Matemática, 25(2), 89-118.

Singletary, L. M, (2012). Mathematical connections made in practice: an examination of teachers' beliefs and practices, [Tesis de doctorado no publicada]. The University of Georgia. United States of America.

Stewart, J., Redlin, L., y Watson, S. (2005). Precálculo. Matemáticas para el Cálculo. Sexta edición. Cengage Learning Editores, S.A.

Trejo, M., y Ferrari, M. (2018). Desarrollo del razonamiento covariacional en estudiantes de nivel medio superior. El caso de la función exponencial. Innovación e Investigación en Matemática Educativa, 3(1), 35-58.

Weber, K. (2002). Students' understanding of exponential and logarithmic functions. En D. Quinney (Ed.), Proceedings of the 2nd international Conference on the Teaching of Mathematics (1-7). John Wiley y Sons.

Yoon, C., Dreyfus, T. y Thomas, M. (2010). How high is the tramping track? mathematising and applying in a calculus model-eliciting activity. Mathematics Education Research Journal, 22(1), 141-157. https://doi.org/10.1007/BF03217571

JaVIER GARCÍA-García

Dirección: Calle Chilpancingo Lote 16 Mza 1 (entre las calles San Marcos e Iguala) Col. Diana Laura Riojas, Chilpancingo de los Bravos, Gro. C.P. 39078

Teléfono: $\quad 7471773357$ 